\title{
What happens in Josephson junctions at high critical current densities
}

\author{
D. Massarotti, D. Stornaiuolo, P. Lucignano, and R. Caruso
}

Dipartimento di Fisica E. Pancini, Universitá di Napoli Federico II, Monte S. Angelo via Cinthia, 80126 Napoli, Italy and CNR-SPIN UOS Napoli, Monte S. Angelo via Cinthia, 80126 Napoli, Italy

\section{Galletti and D. Montemurro}

Department of Microtechnology and Nanoscience, Chalmers University of Technology, SE-412 96 Göteborg, Sweden

\section{B. Jouault}

Laboratoire Charles Coulomb (L2C), UMR 5221 CNRS-Université de Montpellier, F-34095 Montpellier, France

\section{G. Campagnano}

Dipartimento di Fisica E. Pancini, Universitá di Napoli Federico II, Monte S. Angelo via Cinthia, 80126 Napoli, Italy and CNR-SPIN UOS Napoli, Monte S. Angelo via Cinthia, 80126 Napoli, Italy

\section{H. F. Arani}

Dipartimento di Fisica E. Pancini, Universitá di Napoli Federico II, Monte S. Angelo via Cinthia, 80126 Napoli, Italy and Department of Materials Engineering, Engineering Faculty, Imam Khomeini International University, Qazvin, Iran

\section{Longobardi}

American Physical Society, 1 Research Road, Ridge, New York 11961, USA

\section{Parlato and G. P. Pepe}

Dipartimento di Fisica E. Pancini, Universitá di Napoli Federico II, Monte S. Angelo via Cinthia, 80126 Napoli, Italy and CNR-SPIN UOS Napoli, Monte S. Angelo via Cinthia, 80126 Napoli, Italy

\section{G. Rotoli}

Dipartimento di Ingegneria Industriale e dell' Informazione, Università degli Studi della Campania Luigi Vanvitelli, I-81031 Aversa, CE, Italy

\section{A. Tagliacozzo}

Dipartimento di Fisica E. Pancini, Universitá di Napoli Federico II, Monte S. Angelo via Cinthia, 80126 Napoli, Italy and CNR-SPIN UOS Napoli, Monte S. Angelo via Cinthia, 80126 Napoli, Italy

\section{F. Lombardi}

Department of Microtechnology and Nanoscience, Chalmers University of Technology, SE-412 96 Göteborg, Sweden F. Tafuria)

Dipartimento di Fisica E. Pancini, Universitá di Napoli Federico II, Monte S. Angelo via Cinthia, 80126 Napoli, Italy and CNR-SPIN UOS Napoli, Monte S. Angelo via Cinthia, 80126 Napoli, Italy (Submitted March 4, 2017)

Fiz. Nizk. Temp. 43, 1023-1031 (July 2017)

The impressive advances in material science and nanotechnology are more and more promoting the use of exotic barriers and/or superconductors, thus paving the way to new families of Josephson junctions. Semiconducting, ferromagnetic, topological insulator and graphene barriers are leading to unconventional and anomalous aspects of the Josephson coupling, which might be useful to respond to some issues on key problems of solid state physics. However, the complexity of the layout and of the competing physical processes occurring in the junctions is posing novel questions on the interpretation of their phenomenology. We classify some significant behaviors of hybrid and unconventional junctions in terms of their first imprinting, i.e., current-voltage curves, and propose a phenomenological approach to describe some features of junctions characterized by relatively high critical current densities $J_{c}$. Accurate arguments on the distribution of switching currents will provide quantitative criteria to understand physical processes occurring in high- $J_{c}$ junctions. These notions are universal and apply to all kinds of junctions. Published by AIP Publishing.

[http://dx.doi.org/10.1063/1.4995630] 


\section{Introduction}

The novel opportunities offered by nanotechnologies and material science have enlarged the physical conditions of occurrence of the Josephson effect. ${ }^{1,2}$ Still obeying to the general rules given by proximity effect and Andreev reflection, the manner superconductivity propagates along the barrier acquires more and more specific features characteristic of the type of the junction. Specific effects might thus appear, depending on the peculiar geometry/topology or on the material of the devices. Nanotechnology applied to pattern bridges and wires, the ability to integrate nano-wires or almost two-dimensional flakes as barriers in between superconducting pads and grain boundary (GB) junctions have, for instance, favored the use of a coplanar geometry with more complicate layout of the whole device. The various transport channels are playing together and are often activated or filtered by external or intrinsic knobs, and more importantly can be traced through the study of currentvoltage $(I-V)$ characteristics.

In this work we give a comparative analysis of different types of unconventional junctions ranging from high critical temperature superconductors (HTS) GB Josephson junctions (JJs) to hybrid junctions with ferromagnetic or semiconducting barriers, including InAs nanowires and flakes of topological insulators (TI) graphene. We focus on some lack of consistency between experimental measurements and standard models. In particular, we refer to the description of the $I-V$ curves of junctions characterized by high values of the critical current density $J_{c}$ in terms of the resistively shunted junction (RSJ) model. ${ }^{1-4}$ Unconventional junctions renew some inconsistencies, that were clearly detected in the past in traditional trilayer $\mathrm{Nb}$ technology, ${ }^{5,6}$ in a more subtle manner, probably due to the layout of the devices and their intrinsic complexity. We speculate on how higher $J_{c}$ in extended nonuniform barriers or in filaments embedded in an insulating matrix may even promote the generation of a vortex flow, of phase slips events or of heating modes. ${ }^{7,8}$ When possible, this will be done through analysis of the switching distribution of the critical current in hysteretic $I-V$ curves. This comparative investigation contributes to set some benchmarks to discriminate an "authentic" complete Josephson behavior from regimes spoilt by heating mechanisms in high voltage and current ranges.

\section{I-V curves of unconventional junctions}

Figure 1 presents a collection of $I-V$ curves of various unconventional JJs trying to cover several significant regimes and layouts. We report $I-V$ curves of junctions employing two-dimensional barriers as topological insulator flakes [Fig. 1, panels $(\mathrm{a})^{9}$ and $(\mathrm{b})^{10}$ ] or graphene [Fig. 1, panels (e) ${ }^{11,12}$ and (f) ${ }^{13}$ In Fig. 1, panels (c) ${ }^{14}$ and (d), ${ }^{15} I-V$ characteristics refer to junctions where the barrier is a nanowire. In all these cases we present curves with and without hysteresis to encompass nominally the relevant limits in the phase dynamics.

The basis for the understanding of $I-V$ curves is obviously the RSJ model. This model, first introduced by McCumber and Stewart, ${ }^{3,4}$ and later implementations ${ }^{1,2}$ are the main tools to describe $I-V$ phenomenology. Representing the displacement current by a capacitor $C$ and the sum of the quasiparticle and insulator leakage current by a resistance $R$, the well-known equivalent circuit for the junction gives the relation

$$
I=I_{c} \sin \phi+V / R+C d V / d t .
$$

A wide variety of $I-V$ characteristics can be described through an opportune choice of the parameters. We can therefore pass from a tunnel-like behavior with high values of the capacitance, characterized by a hysteretic behavior and by the presence of switching currents, to a regime where capacitance plays a marginal role and no hysteretic behavior is present.

This equation can be transformed in

$$
\left(\frac{\Phi_{0}}{2 \pi}\right)^{2} C \frac{\partial^{2} \phi}{\partial t^{2}}+\left(\frac{\Phi_{0}}{2 \pi}\right)^{2} \frac{1}{R} \frac{\partial \phi}{\partial t}+\frac{\partial}{\partial \phi} U=0,
$$

where

$$
U=-\frac{\Phi_{0}}{2 \pi}\left(I_{c} \cos \phi+I \phi\right)
$$

which is commonly the basis to study the nonlinear dynamics of the junction. This equation describes the motion of a ball moving on the "tilted washboard" potential $U .{ }^{3,4}$ The term involving $C$ represents the mass of the particle, the $1 / R$ term represents the damping of the motion, the average "tilt" of the washboard is proportional to the bias current $I$ and $\Phi_{0}=h / 2 e$ is the flux quantum. Damping is however strongly influenced by the environment, i.e., the circuitry connected to the junction and some aspects will be discussed in the next sections.

For values of $I<I_{c}$, the particle is confined to one of the potential wells, where it oscillates back and forth at the plasma frequency $\left.\omega_{p}=2 \pi I_{c} / \Phi_{0} C\right)^{1 / 2}\left(1-\left(I / I_{c}\right)^{2}\right)^{1 / 4}$.

The McCumber-Stewart damping parameter $\beta_{c}=2 \pi I_{c}$ $R^{2} C / \Phi_{0}^{2}$ determines the amount of damping. ${ }^{1,2}$ The strength of the friction can be also expressed through the junction quality factor $Q=\omega_{p} R C$. In a more general approach, $Q$ has a frequency dependence, ${ }^{16}$ which includes the effects of the external shunting impedance. Junctions are underdamped, with hysteretic $I-V$ curves, and hence latching for $\beta_{c}>1$. For $\beta_{c}<1$ they are over-damped, with nonhysteretic $I-V$, and nonlatching.

In Fig. 1(a) $I-V$ curves of a Al-BiSe (flake) (TI)-Al junction as a function of the temperature clearly indicate an overdamped regime, ${ }^{9}$ which is retrieved in Fig. 1(c) for a Al-InAs (nanowire)-Al device. ${ }^{14}$ In these devices, the value of the critical current density $J_{c}$ is strongly affected by the properties of the interface. ${ }^{17}$ In these systems a nominal value of the critical current density per unit length $W$ ( $W$ being the width of the junction) $J_{c w}=10^{-3} \mathrm{~A} / \mathrm{cm}$ for the $\mathrm{Al}-\mathrm{BiSe}-\mathrm{Al}$ junction and of the standard $J_{c}=10^{3} \mathrm{~A} / \mathrm{cm}^{2}$ (normalized to the cross section of the nanowire) for $\mathrm{Al}-\mathrm{InAs}-\mathrm{Al}$ junction can be estimated, respectively. In this case the InAs nanowire is placed on the top of $\mathrm{Al}$ banks, differently from the standard configuration where the $\mathrm{Al}$ is rather deposited on the nanowire. ${ }^{15}$ This design circumvents the compatibility problems stemming from the peculiar growth condition requirements of some materials, as for instance HTS, and its coupling with special barriers. ${ }^{18}$ In this layout, the integration of the barrier with the 

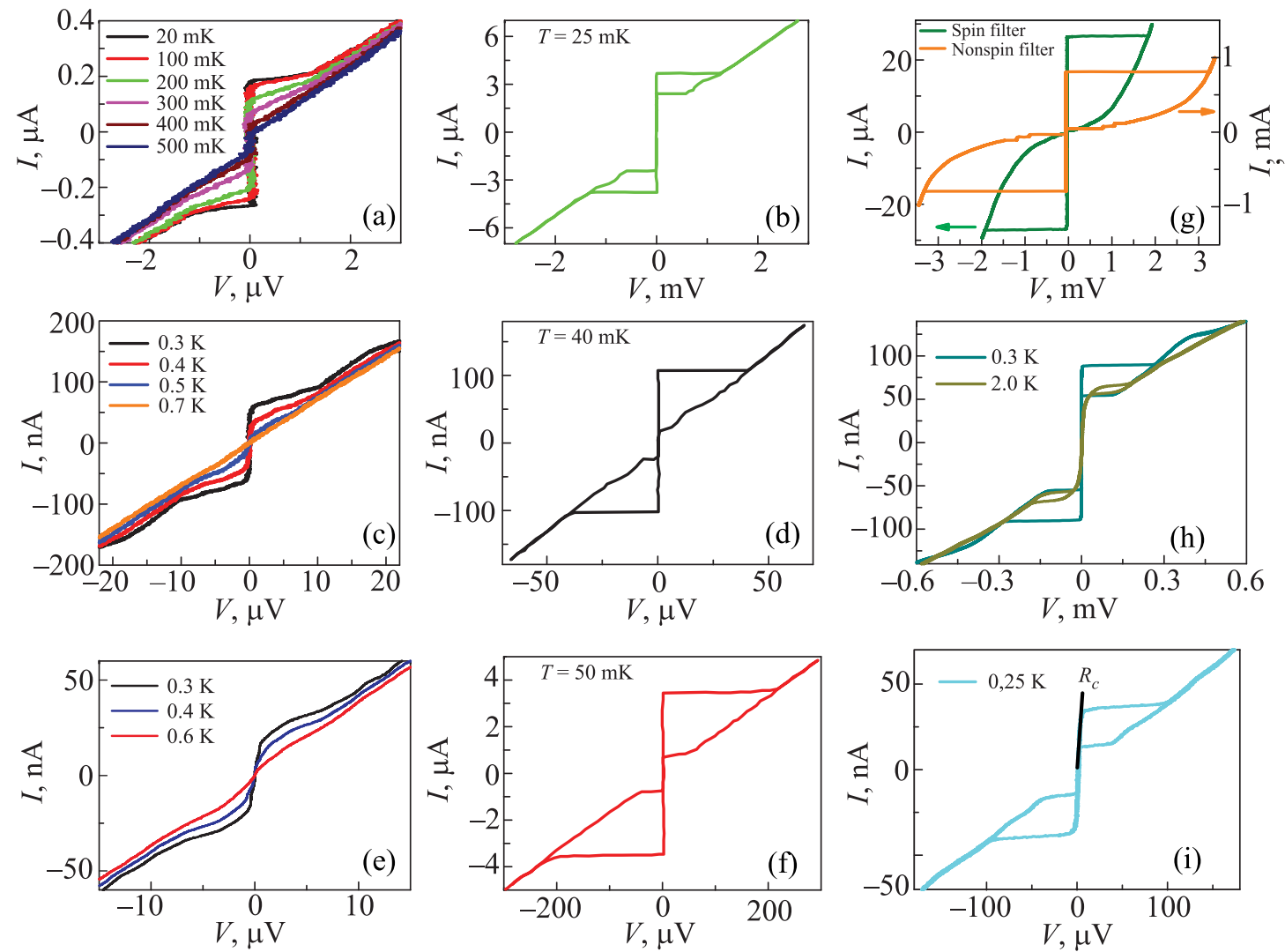

Fig. 1. Measurements of $I-V$ characteristics on different types of unconventional JJs. In panels (a) and (b) $I-V$ curves refer to TI JJs, Al-BiSe (flake)-Al junctions ${ }^{9}$ in (a) and $\mathrm{Nb}-$ strained bulk $\mathrm{HgTe}-\mathrm{Nb}$ (adapted from Ref. 10) in (b), respectively. In panels (c) and (d) $I-V$ characteristics refer to on Al-InAs (nanowire)-Al are reported, adapted from Ref. 4 in (c) and from Ref. 15 in (d), respectively. Panels (e) and (f) show typical $I-V$ curves of graphene-based JJs, in particular Al-graphene-Al JJ ${ }^{11,12}$ in (e) and PbIn-graphene-PbIn in (f) (adapted from Ref. 13). In all the three rows, the panel on the left reports the temperature dependence of nonhysteretic $I-V$ characteristics, while the central panel shows the case of hysteretic $I-V$ curves, respectively. In panel (g) the $I-V$ curves refer to ferromagnetic $\mathrm{NbN}-\mathrm{GdN}-\mathrm{NbN}$ spin filter junctions with different values of $J_{c}{ }^{21}$ and finally panel (h) and panel (i) show the $I-V$ characteristics of YBCO biepitaxial GB junctions with $J_{c}$ of $65 \mathrm{~A} / \mathrm{cm}^{2}$ and $5 \mathrm{~A} / \mathrm{cm}^{2}$, respectively. ${ }^{25}$ The black line in panel (i) indicates the finite slope $R_{0}$ of the supercurrent branch (see the text).

super-conducting components takes place at room temperature, after suitable surface treatments assembling optimally pre-built blocks. The nanowire (NW) is suspended on the superconducting electrodes with $\mathrm{Ti} / \mathrm{Au}$ contacts encapsulating the InAs-NW edges. ${ }^{19}$

The curves relative to the Al-graphene (sheet)-Al JJ, reported in Fig. 1(e) as a function of temperature, also point to overdamped behavior. ${ }^{11,12}$ Here $J_{c w}=10^{-4} \mathrm{~A} / \mathrm{cm}$. In the case of graphene barrier the supercurrent is superimposed on a small resistance, which has been correlated to an incipient Berezinskii-Kosterlitz-Thouless (BKT) transition. ${ }^{11}$ Vortex bundles break above the BKT temperature and move in the barrier adding a resistive channel. This is a direct consequence of the layout of the junction with an extremely large graphene sheet with an almost ideal two-dimensional behavior. The barrier can host very extended vortex bundles and give them space to move, which results in an additional intrinsic dissipation mechanism. This is an example of the generation of intrinsic dissipation mechanism not necessarily due to high $J_{c}$ passing thorough the junction.

Hysteretic $I-V$ curves are reported for the following junctions: Nb-strained bulk HgTe (TI)-Nb [Fig. 1(b)], ${ }^{10} \mathrm{Al}-\mathrm{InAs}$ (nanowire)-Al [Fig. 1(d)] ${ }^{15}$ and $\mathrm{PbIn-graphene-PbIn} \mathrm{[Fig.}$ 1(f).$^{13}$ The nominal values of $J_{c w}$ and $J_{c}$ are $10^{-2} \mathrm{~A} / \mathrm{cm}, 5$ $\times 10^{3} \mathrm{~A} / \mathrm{cm}^{2}$ and $10^{-3} \mathrm{~A} / \mathrm{cm}$, respectively. In literature there are several other examples with similar $I-V$ curves. For most of these curves there is no exact fitting with RSJ predictions and no reliable values of the capacitance are extracted. These inconsistencies seem to raise doubts about a truly underdamped behavior, that one would naively expect on the basis of RSJ arguments in presence of hysteretic $I-V$ curves.

This cannot be even explained with the extension of the RSJ model, ${ }^{2}$ which includes other possible dissipation mechanisms occurring in the subgap region and manifesting themselves through characteristic leakage currents. These are identified as nonlinear resistive models. The term $I_{N}=V / R$ is replaced by

$$
I_{N}(V)=V \begin{cases}1 / R_{L} & \text { for }|V|<V_{g} \\ 1 / R_{n} & \text { for }|V|>V_{g}\end{cases}
$$

or by a more reasonable approximation of the power-law

$$
I_{N}(V)=\frac{V}{R_{n}} \frac{\left(V / V_{g}\right)^{n}}{1+\left(V / V_{g}\right)^{n}} .
$$

In Eqs. (4) and (5), being $V_{g}=\left|\left(\Delta_{1}+\Delta_{2}\right) / e\right|, \Delta_{1}$ and $\Delta_{2}$ the gap of the two superconducting electrodes, while $R_{L}$ and $R_{n}$ are the subgap quasiparticle resistance and the normal state resistance, respectively. These versions of the RSJ model have the merit of modeling leakage currents for voltages lower than the sum of the gap values $V_{g}$ of the superconductors composing the junction. $V_{g}$ is commonly assumed about the sum of the gap values, but in general this 
voltage value, to which the current switches in nominally underdamped junctions, can be quite different, introducing a substantially new scaling energy other than $V_{g}$.

We complete our overview on typical $I-V$ curves of unconventional systems by illustrating ferromagnetic and HTS JJs, which provide additional elements of reference.

The $I-V$ curves in Fig. $1(\mathrm{~g})$ refer to ferromagnetic $\mathrm{NbN}-\mathrm{GdN}-\mathrm{NbN}$ junctions with $J_{c}$ in the range between 50 and $10^{3} \mathrm{~A} / \mathrm{cm}^{2}$, and cover the spin filter and non-spin filter regimes, respectively. ${ }^{20,21}$ These junctions are classical trilayers, where unconventional behaviors all come from the ferro-insulator barriers of GdN. These are among the very few ferromagnetic junctions displaying underdamped behavior, as opposed to all other ferromagnetic junctions falling in the overdamped regime. In the low- $J_{c}$ case the evidence of macroscopic quantum tunneling gives a very accurate way to evaluate junction parameters, in particular the effective damping and the capacitance in the framework of the RSJ model. ${ }^{21}$ Common to most of junctions with hysteretic $I-V$ curves discussed up to now, including the latest ferromagnetic junctions, is the discrepancy between the expected latching voltage value $\left(V_{s w}\right)$ after the switch of the critical current from the superconducting state and the expected value $V_{g}$.

Unconventional behaviors of HTS JJs have been widely discussed in literature (most references can be found in the reviews ${ }^{18,22-24}$ ) and cover a large variety of issues, which are beyond the scope of this manuscript. We confine our interest to specific aspects related to $I-V$ curves. HTS JJs are fundamental reference systems despite their complexity, because they span a wide range of junction parameters, as for instance $J_{c}$, the specific resistance $R_{n} A$, where $A$ is the cross section, and because their characteristic energies can be also scaled over three orders of magnitude.

Figure 1, panels (h) and (i), refer to YBCO biepitaxial GB junctions with $J_{c}$ of 65 and $5 \mathrm{~A} / \mathrm{cm}^{2}$, respectively. ${ }^{25}$ The $I-V$ curves are highly hysteretic, with a difference between the critical and the retrapping current up to $70 \%$ at $300 \mathrm{mK}$. The small dimensions of these devices (width $w=600 \mathrm{~nm}$ ) are expected to reduce the influence of micro-structural defects in the junctions properties. As a consequence, there is a good correspondence between the switching voltage $V_{s w}$ and the $I_{c} R_{n}$ product, ${ }^{26,27}$ where $R_{n}$ is the normal state resistance of the junction. These curves give clear benchmarks for the low- $I_{c}$ limit, completing all known extensions of the RSJ model. Interestingly, hysteresis and phase diffusion coexist in the $I-V$ curves. In order to account for this coexistence, a modified RSJ model is required, including a frequency dependent damping. ${ }^{16,25,28,29}$

These two phenomena usually arise in different parameter ranges of the RSJ model. Their coexistence in the same $I-V$ is therefore unusual ${ }^{28-30}$ and can be only understood with a finer analysis of the devices dynamics. We included in the RSJ model an additional quality factor $Q_{1}$ in order to take into account the contribution of the circuit the junction is embedded into. According to the "tilted washboard" potential model, at low voltage the phase particle oscillates at the plasma frequency, typically in the gigahertz range. In this case, the smaller quality factor $Q_{1}$ dominates the behavior of the whole system. The voltage state involving steady motion of the phase is instead dominated by the higher quality factor $Q$. Therefore, the system will exhibit a different damping depending on the voltage (frequency) range. ${ }^{25}$

If $I_{c}$ is further reduced, phase delocalization effects also have to be included in this picture. ${ }^{29}$ Figure 1(i) shows the $I-V$ curve of an HTS junction with $J_{c}=5 \mathrm{~A} / \mathrm{cm}^{2}$. In this case, the value of the Josephson energy $E_{J}=\hbar I_{0} / 2 e$ (where $I_{0}$ is the zero temperature critical current) is greatly reduced, becoming comparable to that of the Coulomb energy $E_{c}=e^{2} / 2 C . E_{c}$ therefore cannot be disregarded in the analysis of the junctions dynamics, leading to phase delocalization effects. For values of $x=E_{c} / E_{J}$ greater than 0.25 phase delocalization leads to an increase in the probability for the phase to escape from the potential well, both in the thermal and in the quantum regimes. Multiple escapes and retrapping events result in the appearance of a finite resistance $R_{0}$ at low voltage [see black line in Fig. 1(i)].

Table 1 condenses parameters for additional hybrid junctions taken from literature ${ }^{31-35}$ and from the examples above.

\section{Processes occurring in junctions with high $J_{c}$}

In this section we describe two different effects occurring in high- $J_{c}$ JJs. The former refers to the possible occurrence of vortex motion, with the appearance of a characteristic bending in the $I-V$ curves at certain voltage values. The latter is more subtle and is related to the appearance of heating modes, manifesting in switching current distributions.

\subsection{What might happen in the $I-V$ curves}

In Fig. 2 we show the $I-V$ characteristics of a YBCO biepitaxial $\mathrm{JJ}$ characterized by high values of $J_{c} \approx 10^{5} \mathrm{~A} /$ $\mathrm{cm}^{2}$ and relatively high values of $I_{c}$, when compared to the values of the junctions reported in Fig. 1. The $I-V$ curve is hysteretic but we are more interested in the high voltage behavior. After the switch from the superconducting to the normal state, the first upward (1st) bending in the $I-V$ curve is consistent with a RSJ-like behavior. The second downward (2nd) bending is not consistent with RSJ behavior. We

TABLE 1. Properties of different types of hybrid Josephson junctions. $L$ represents the length of the nanowire or the distance between the superconducting electrodes or the thickness of the ferromagnetic-insulator barrier or the width of the YBCO GB JJ, depending on the type of the junction, respectively.

\begin{tabular}{lcccc}
\hline \hline Type of junction & $L, \mathrm{~nm}$ & $\begin{array}{c}I_{c}, \mathrm{nA} \\
(\text { at } T, \mathrm{mK})\end{array}$ & $\begin{array}{c}I_{c} R_{n}, \\
\mu \mathrm{V}\end{array}$ & References \\
\hline Nb-InN (NW)-Nb & $\approx 100$ & $5700(800)$ & 450 & 31 \\
Al-InAs (NW)-Al & from 100 to 450 & $130(40)$ & $2-60$ & 15 \\
Al-InAs (NW)-Al & 140 & $60(300)$ & 10 & 14 \\
Al-GeSi-Al & $\approx 100$ & $120(60)$ & 200 & 32 \\
Al-graphene-Al & $\approx 400$ & $35(30)$ & 120 & 33 \\
Al-graphene-Al & $\approx 400$ & $500(60)$ & 50 & 34 \\
Al-graphene-Al & $\approx 200$ & $50(300)$ & 15 & 11 \\
PbIn-graphene-PbIn & $\approx 300$ & $1000(50)$ & 200 & 13 \\
Nb-Bi 2 Te ${ }_{3}-\mathrm{Nb}$ & $\approx 50$ & $18 \mu \mathrm{A}(260)$ & 20 & 35 \\
Al-Bi ${ }_{2} \mathrm{Se}{ }_{3}-\mathrm{Al}$ & $\approx 300-400$ & $230-1700(300)$ & $10-90$ & 9 \\
Nb-HgTe-Nb & $\approx 200$ & $3.8 \mu \mathrm{A}(25)$ & 200 & 10 \\
NbN-GdN-NbN & 3.0 & $30 \mu \mathrm{A}(300)$ & 100 & 21 \\
NbN-GdN-NbN & 1.5 & $820 \mu \mathrm{A}(300)$ & 1000 & 21 \\
YBCO GB & 500 & $100(300)$ & 600 & 25 \\
YBCO GB & 600 & $5(300)$ & 60 & 25 \\
\hline \hline
\end{tabular}


propose an explanation in terms of vortex motion. Our analysis of this $I-V$ curve is based on the fact that the voltage across the junction at $I>I_{c}$ comes from two different mechanisms: $V=V_{R S J}+V_{\text {vor }}$, where the first contribution (dominant at low energies) comes from the conventional RSJ Josephson dynamics, whereas the second, dominating at high energies, comes from vortex dynamics. The latter does not carry any phase information, being completely unrelated to the Josephson component, but it is rather controlled by flux-line dynamical effects. It includes thermal effects, depinning, creep and flow contribution. ${ }^{5,6,36}$ This commonly applies to standard bridges without Josephson coupling. ${ }^{5,6}$ In Fig. 2 we report two measurements at $T \ll T_{c}$, namely $T=340 \mathrm{mK}$ and $3 \mathrm{~K}$ (blue and red points, respectively). Here, neglecting the jump at $I_{c}$, the RSJ contribution can be approximated to

$$
V_{R S J}=I_{c 1} R_{n} \sqrt{1-\left(I / I_{c 1}\right)^{2}}
$$

where $I_{c 1}$ is the Josephson critical current. When the current increases, vortex motion perpendicular to the current can give rise to

$$
V_{\text {vor }}=V_{0} \exp \left(-U_{0} /\left(k_{B} T\right)\right) \sinh \left(U_{0} /\left(k_{B} T\right) I / I_{c 2}\right),
$$

where $I_{c 2}$ is a second critical current regulating the vortex motion, as well as $U_{0}$ that is the classical activation barrier for vortices. ${ }^{5,6}$ According to the critical state model, ${ }^{6,37}$ one expects $U_{0} /\left(k_{B} T\right) \sim 4 \pi \nu$ with $\nu=1$ While the $I_{c 1} R_{n}$ parameter $(\sim 0.9 \mathrm{mV}$ at $340 \mathrm{mK}, \sim 0.85 \mathrm{mV}$ at $3 \mathrm{~K})$ is fixed by the energy scales, the substantial free parameters are $V_{0} \sim 140$ $\mu \mathrm{V}$ and $I_{c 2} \sim 20 \% I_{c 1}$. The results of the fits are reported in Fig. 2 (blue and red lines for $T=340 \mathrm{mK}$ and $T=3 \mathrm{~K}$, respectively). The physical interpretation of this phenomenological approach is quite direct. The lack of uniformity is modeled through the presence of a nanochannel, some kind of filamentary structure. The condition $I_{c 2} \sim 20 \% I_{c 1}$ implies that the nanochannel is much smaller that the total area of the junction, otherwise its contribution would be dominant. The nanochannel triggers vortex flow on the scale energy of $U_{0}$ inside the slab which contains the GB. No significant variations between $300 \mathrm{mK}$ and $3 \mathrm{~K}$ are observed, since the

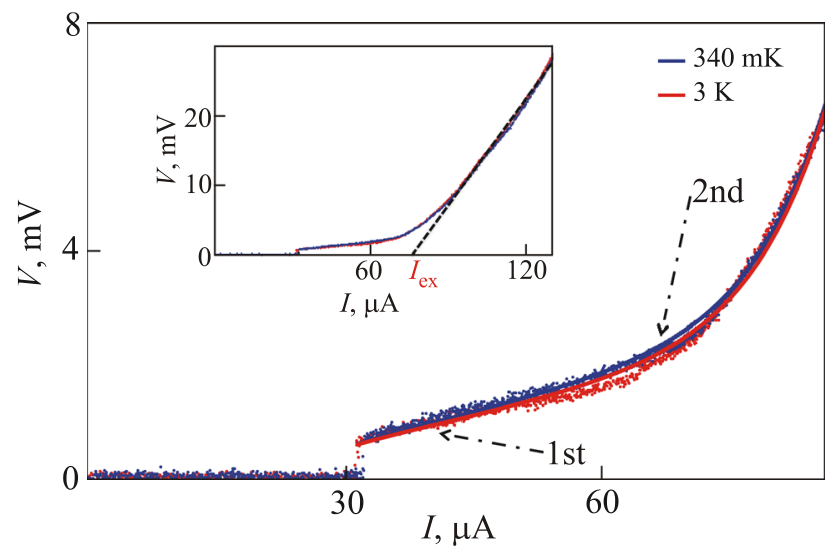

Fig. 2. $I-V$ characteristics measured on high- $J_{c}$ GB JJ (blue and red points refer to measurements at $340 \mathrm{mK}$ and $3 \mathrm{~K}$, respectively) are fitted according to Eqs. (6) and (7), which take into account both the RSJ model and vortex dynamics (blue and red line at $340 \mathrm{mK}$ and $3 \mathrm{~K}$, respectively). The inset shows the same $I-V$ curves in a wider range of current and voltage. energy scale of the activation vortex motion is much higher. This shape of the $I-V$ curves would naturally lead to an alternative explanation of the excess current $I_{\mathrm{ex}}$, as strongly influenced by the activated vortex motion. Similar bumps might be also induced by time dependent effects included in the Ginzburg-Landau equations, whose application would be less direct in this case. ${ }^{2}$ These $I-V$ curves are clearly different from those measured in simple nanowires (see for instance Ref. 38 and references therein).

\subsection{Heating modes and nonequilibrium in switching current measurements}

The washboard potential offers a very intuitive picture to understand thermally activated processes and macroscopic quantum phenomena. ${ }^{39}$ Measurements of switching current distributions (SCDs), along with their first and second momenta (the mean $\bar{I}$ and the width $\sigma$ ), codify the very general process of the escape of a particle (phase) from a potential well in a JJ. ${ }^{1,2}$ Roughly speaking, SCDs are obtained in JJs with hysteretic $I-V$ characteristics by measuring the current at which the transition from the zero voltage state to the finite voltage state occurs. The stochastic nature of this process can be studied by repeating the measurement many times, typically $10^{4}$ times, and the collection of all the events provides the switching current distribution.

Thermally activated processes are well understood in JJs both in the underdamped ${ }^{40-42}$ and in the moderately damped ${ }^{16}$ regime. The transition to the macroscopic quantum tunneling (MQT) regime has been theoretically ${ }^{39,43}$ and experimentally ${ }^{44-46}$ widely investigated. In moderately damped junctions, since dissipation levels are larger, the phase particle after the escape event can be retrapped in one of the following wells of the washboard potential. This dynamics generates a diffusive motion of the phase particle, namely a phase diffusion process, ${ }^{47-51}$ whose fingerprint is the collapse of $\sigma$ above a transition temperature $T^{*}$. In panels (a) and (b) of Fig. 3 an example of the temperature behavior of the switching distributions and of $\sigma(T)$, respectively, typical of moderately damped junctions is shown.

The SCDs measured on high- $J_{c}$ GB junction is reported in panel (c) of Fig. 3, along with the corresponding temperature behavior of $\sigma$ in panel (d) of the same figure. The phase dynamics is radically different from what observed in standard junctions with low- $J_{c}$ values. The rate of decrease of $\sigma$ [panel (d) of Fig. 3] above the transition temperature $T^{*}$ turns to be a distinctive marker of the phase dynamics, since the slope is much smaller when compared to moderately damped JJs, and clearly indicates that the phase dynamics of high- $J_{c}$ JJs cannot be described in terms of the intermediate dissipation regime. ${ }^{52}$

Therefore, hysteresis in $I-V$ curves $^{15,53}$ does not necessarily indicate canonical Josephson phase dynamics, even in the presence of a Fraunhofer magnetic field pattern. ${ }^{8}$ It may rather arise as a result of local heating processes, possibly induced by intrinsic inhomogeneous composition unavoidable for high- $J_{c}$ junctions. The absence of a set of selfconsistent electrodynamics parameters to describe high- $J_{c} \mathrm{JJ}$ is a strong indication of the failure of the standard Josephson dynamics. This failure is of general relevance, applying both to conventional low- $T_{c}$ superconductor (LTS) $\mathrm{JJs}^{7,8}$ and to the emergent class of hybrid nanoscale junctions. ${ }^{15,33,34}$ 
We have found that the numerical simulation of a transition driven by local heating events accounts well for devices in the $J_{c}$ interval $\left(10^{4}-10^{5} \mathrm{~A} / \mathrm{cm}^{2}\right) .{ }^{52}$ For larger values of $J_{c}$, heating driven mechanisms become dominant with a transition to the normal state locally in the junction area. These events can be modeled as phase slips events (PSEs), in the sense that they are local processes, break the coherence of the phase information and are described by a heat diffusion-like equation. In particular, the probability for a single heating event can be still described in terms of the Langer-Ambegaokar-McCumber-Halperin (LAMH) theory $^{54,55}$ and further extensions. ${ }^{56}$

Following an approach proposed in Ref. 57 for LTS wires, our numerical simulation of the temperature jump induced by a PSE obeys the phenomenological diffusive equation for the relaxation of the temperature gradient

$$
\frac{d \delta T}{d t}+\alpha\left(T, T_{b}\right) \delta T=r\left(T_{b}, t\right)+\eta(T, I) \sum_{i} \delta\left(t-t_{i}\right) .
$$

Here $\delta T=T-T_{b}$ is the deviation from the bath temperature $T_{b}$. The relaxation coefficient $\alpha\left(T, T_{b}\right)$ depends on the thermal conductivity $K(T)$ and on $T_{b} \cdot r\left(T_{b}, t\right)$ is the noise source due to the environment with an admittance $Y(\omega)$, while $\eta(T, I)$ is the temperature jump due to the PSEs, which occur at the stochastically distributed times $t_{i}$. After the heating event, the transition to the finite voltage state occurs if the local temperature of the junction overcomes a threshold temperature $T_{\mathrm{th}}$. More details can be found in Ref. 50 .
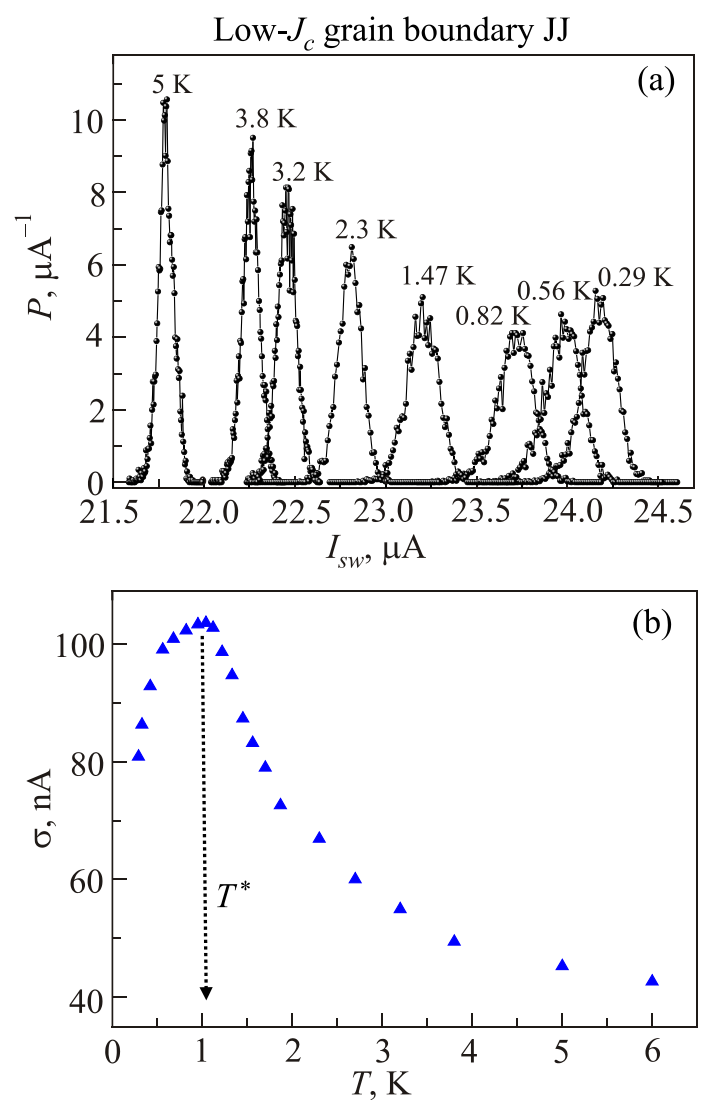

Two main effects discriminate between the lowtemperature and the high-temperature behavior. At low temperatures the specific heat is quite low, thus with each PSE there is a considerable increase in the temperature. In addition, the thermal conductivity is quite low as the system is deeply into the superconducting phase. The junction is rather isolated from the environment and the temperature jump due to a single heating event is destructive for the superconducting state. A large local heating is produced which is difficult to dissipate. Therefore, the system is not at equilibrium with its environment, and we can define an effective temperature $T_{\text {eff }}$ for the junction, which is higher than $T_{b}$.

At high temperatures we are in the opposite regime of small $\eta(T)$ per heating event, while the thermal conductivity $K(T)$ increases with increasing temperature as well. Thermal diffusion is more effective and multiple PSEs are required for switching. This occurs above $T^{*}$, where the derivative $d \sigma / d T$ is negative. It can be shown that $T_{\text {eff }}$ and $T_{b}$ coincide above $T^{*}$, since the system is able to thermalize during the time interval between well separated heating events. In this temperature range, the number of successive PSEs, which are responsible for the transition, can be estimated. A consistent set of the junction parameters (temperature jump $\eta$, number of heating events) can be extracted from these simulations. $^{52}$

The final result is that a Josephson junction cannot sustain an unlimited increase in the critical current $I_{c}$, and thus in the quality factor $Q$, through larger critical current density
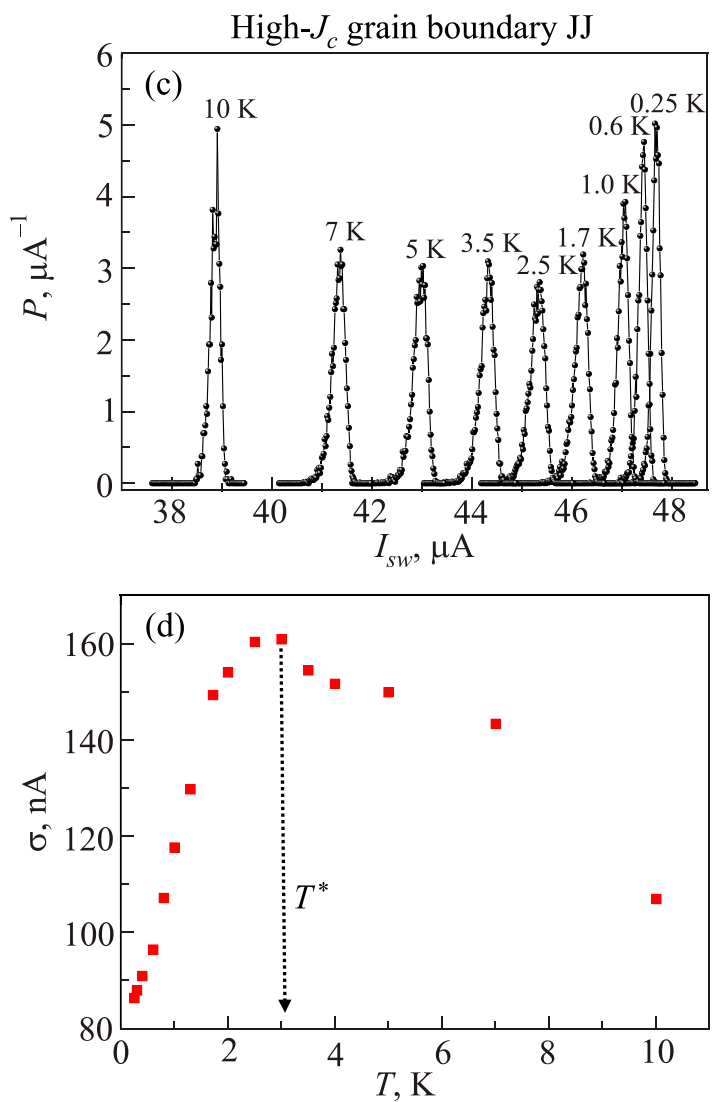

Fig. 3. (a) Measurements of SCDs on low- $J_{c}$ moderately damped GB JJ. The corresponding $\sigma(T)$ is reported in panel (b). The transition temperature $T^{*}$ indicates the onset of the phase diffusion regime. In panel (c) the measurements on high- $J_{c}$ GB JJ are shown. The switching profiles present evident deviations from the typical temperature behavior of moderately damped JJs, as discussed in the text, signaling the emergence of different dissipation mechanisms in the switching dynamics. The temperature behavior of $\sigma$ in panel (d) is a distinctive marker of local heating events occurring in high- $J_{c}$ JJs. 
$J_{c}$ while still preserving all the properties of the Josephson effect and all the features of the underdamped regime in the $I-V$ curves. The classical Josephson phase dynamics, which takes place in junctions characterized by lower critical current densities $J_{c}$, is replaced at high- $J_{c}$ values by a regime driven by local heating events where phase information is lost. Nonequilibrium phenomena produce hysteretic $I-V$ characteristics and modify the influence of dissipation, thus becoming measurable through modeling of the SCD in terms of heating modes. The transition from classical to nonequilibrium phase dynamics has been found for HTS GB junctions, ${ }^{52}$ but the features of the transition are universal. Specific thresholds may depend on the type of junctions and materials $^{7,8}$

\subsection{Capacitance in high- $J_{c} J J s$}

The effects discussed in the previous section have some consequences on the evaluation of the capacitance in high- $J_{c}$ junctions, that we analyze for HTS JJs. Reported $C / A$ values typically range between $10^{-14}$ and $10^{-12} \mathrm{~F} / \mu \mathrm{m}^{2}$ for a variety of GBs differing in structure, configuration, and misorientation. A possible correlation between $C / A$ and $J_{c}{ }^{18,23,58-60}$ is indicated by the yellow line in Fig. 4(b). A comparative study of the phase dynamics of biepitaxial JJs on STO and LSAT substrates, ${ }^{25-27,51}$ confirms the effects of the stray capacitance of the STO substrate. ${ }^{58-60}$ These experiments ${ }^{25,51}$ use SCD measurements for a more sophisticated estimate of the effective $C$ and have given a more quantitative account of the effects of nonequilibrium heating mechanisms in high- $J_{c}$ junctions. ${ }^{52}$ The relation between $C$ and $J_{c}$ might be more subtle and more questionable at high- $J_{c}$ values. The $C / A$ drop as a function of the $R_{n} A$ product over about three orders of magnitude [orange curve in Fig. 4(a)] ${ }^{58-61}$ seems to be more universal and robust. Data inferred from SCD measurements confirm the trend, as far as $R_{n} A$ values do not correspond to junctions with very high $J_{c}$. More subtle issues on the effective dissipation and $R_{n}$ values can be incorporated. ${ }^{50}$

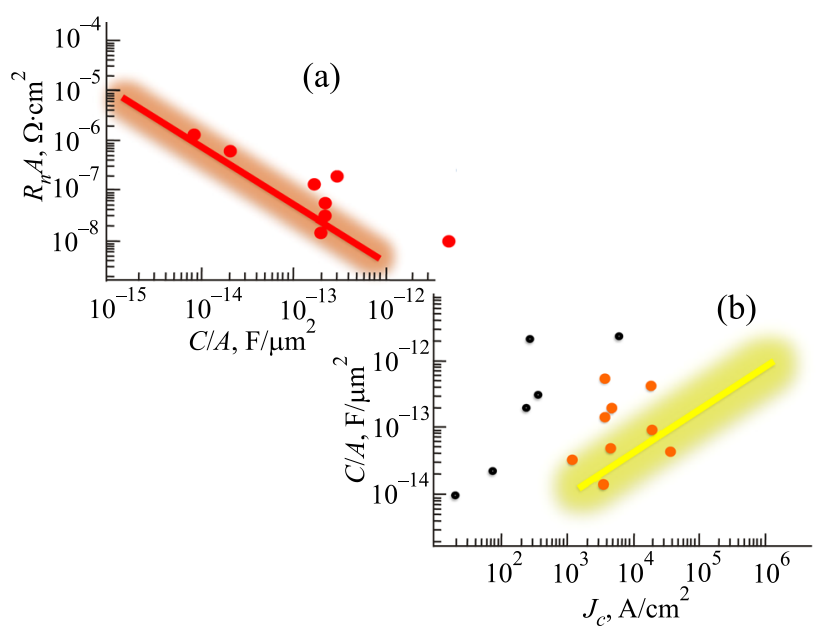

Fig. 4. (a) The orange curve gives the qualitative dependence of $R_{n} A$ on $C /$ $A^{23,60,61}$ for different types of HTS JJs. The red points come from SCD measurements on biepitaxial JJs. ${ }^{25,46,51}$ (b) $C / A$ vs $J_{c}$, the yellow curve represents data collected from literature. ${ }^{23,58-60}$ Orange dots come from Refs. 26 and 27 , while $C / A$ values indicated by black dots are obtained through SCD measurements from Refs. 25, 46, and 51.

\section{Conclusions}

We have analyzed the current-voltage characteristics of different types of unconventional JJs, including HTS GB junctions and hybrid JJs. Different dissipation sources may arise, thus distinctive criteria to distinguish the possible dissipation mechanisms provide the key tool to reconstruct the electrodynamics of such unconventional JJs. The case of high critical current density $J_{c}$ junctions is quite relevant, since in this regime the standard Josephson phase dynamics of a hysteretic junction collapses. In these devices, characterized by intrinsic lack of homogeneity and by inline layout, at high-voltages (from 5 to 10 times $V_{s w}$ ) $I-V$ characteristics may present bumps signatures of activated vortex motion. The analysis of the escape dynamics through measurements of SCDs reveal that different switching profiles occur for high- $J_{c}$ junctions, which can be modeled in terms of local heating events and nonequilbrium phenomena. These effects are of relevance for all the experiments using lowdimensional barriers, for which possible heating effects could lead to distorted phase information.

We acknowledge helpful discussions with T. Bauch, M. G. Blamire, S. Charpentier, A. Pal, and S. Roddaro.

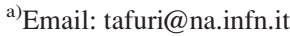

\footnotetext{
${ }^{1}$ A. Barone and G. Paternò, Physics and Applications of the Josephson Effect (John Wiley \& Sons, New York, 1982).

${ }^{2}$ K. K. Likharev, Dynamics of Josephson Junctions and Circuits (Gordon and Breach, New York, 1986).

${ }^{3}$ W. C. Stewart, Appl. Phys. Lett. 12, 277 (1968).

${ }^{4}$ D. E. McCumber, J. Appl. Phys. 39, 3113 (1968).

${ }^{5}$ K. Fossheim and A. Sudbo, Superconductivity: Physics and Applications (John Wiley \& Sons, New York, 2005).

${ }^{6} \mathrm{M}$. Tinkham, Introduction to Superconductivity, 2nd ed. (Dover Publications, 2004)

${ }^{7}$ A. W. Kleinsasser and R. A. Buhrman, Appl. Phys. Lett. 37, 841 (1980).

${ }^{8}$ R. E. Miller, W. H. Mallison, A. W. Kleinsasser, K. A. Delin, and E. M. Macedo, Appl. Phys. Lett. 63, 10 (1993).

${ }^{9}$ L. Galletti, S. Charpentier, M. Iavarone, P. Lucignano, D. Massarotti, R. Arpaia, K. Kadowaki, T. Bauch, A. Tagliacozzo, F. Tafuri, and F. Lombardi, Phys. Rev. B 89, 134512 (2014).

${ }^{10}$ J. B. Oostinga, L. Maier, P. Schüffelgen, D. Knott, C. Ames, C. Brüne, G. Tkachov, H. Buhmann, and L. W. Molenkamp, Phys. Rev. X 3, 021007 (2013).

${ }^{11}$ D. Massarotti, B. Jouault, V. Rouco, S. Charpentier, T. Bauch, A. Michon, A. De Candia, P. Lucignano, F. Lombardi, F. Tafuri, and A. Tagliacozzo, Phys. Rev. B 94, 054525 (2016).

${ }^{12}$ B. Jouault, S. Charpentier, D. Massarotti, A. Michon, M. Paillet, J.-R. Huntzinger, A. Tiberj, A. Zahab, T. Bauch, P. Lucignano, A. Tagliacozzo, F. Lombardi, and F. Tafuri, J. Supercond. Novel Magn. 29, 1145 (2016).

${ }^{13}$ G.-H. Lee, D. Jeong, J.-H. Choi, Y.-J. Doh, and H.-J. Lee, Phys. Rev. Lett. 107, 146605 (2011).

${ }^{14}$ D. Montemurro, D. Stornaiuolo, D. Massarotti, D. Ercolani, L. Sorba, F. Beltram, F. Tafuri, and S. Roddaro, Nanotechnology 26, 385302 (2015).

${ }^{15}$ Y. J. Doh, J. A. van Dam, A. L. Roest, E. P. A. M. Bakkers, L. P. Kouwenhoven, and S. De Franceschi, Science 309, 272 (2005).

${ }^{16}$ R. L. Kautz and J. M. Martinis, Phys. Rev. B 42, 9903 (1990).

${ }^{17}$ L. Galletti, S. Charpentier, Y. Song, D. Golubev, S. M. Wang, T. Bauch, and F. Lombardi, IEEE Trans. Appl. Supercond. 27, 4 (2017).

${ }^{18}$ F. Tafuri and J. R. Kirtley, Rep. Prog. Phys. 68, 2573 (2005).

${ }^{19}$ D. Montemurro, D. Massarotti, P. Lucignano, S. Roddaro, D. Stornaiuolo, D. Ercolani, L. Sorba, A. Tagliacozzo, F. Beltram, and F. Tafuri, J. Supercond. Novel Magn. 28, 3429 (2015).

${ }^{20}$ K. Senapati, M. G. Blamire, and Z. H. Barber, Nat. Mater. 10, 849 (2011).

${ }^{21}$ D. Massarotti, A. Pal, G. Rotoli, L. Longobardi, M. G. Blamire, and F. Tafuri, Nat. Commun. 6, 7376 (2015).

${ }^{22}$ R. Gross, L. Alff, A. Beck, O. M. Froehlich, D. Koelle, and A. Marx, IEEE Trans. Appl. Supercond. 7, 2929 (1997).

${ }^{23}$ H. Hilgenkamp and J. Mannhart, Rev. Mod. Phys. 74, 485 (2002).
} 
${ }^{24}$ F. Tafuri, D. Massarotti, L. Galletti, D. Stornaiuolo, D. Montemurro, L. Longobardi, P. Lucignano, G. Rotoli, G. P. Pepe, A. Tagliacozzo, and F. Lombardi, J. Supercond. Nov. Magn. 26, 21 (2013).

${ }^{25}$ D. Stornaiuolo, G. Rotoli, D. Massarotti, F. Carillo, L. Longobardi, F. Beltram, and F. Tafuri, Phys. Rev. B 87, 134517 (2013).

${ }^{26}$ D. Stornaiuolo, G. Rotoli, K. Cedergren, D. Born, T. Bauch, F. Lombardi, and F. Tafuri, J. Appl. Phys. 107, 113901 (2010).

${ }^{27}$ D. Stornaiuolo, G. Papari, N. Cennamo, F. Carillo, L. Longobardi, D. Massarotti, A. Barone, and F. Tafuri, Super-cond. Sci. Technol. 24, 045008 (2011).

${ }^{28}$ J. M. Martinis and R. L. Kautz, Phys. Rev. Lett. 63, 1507 (1989).

${ }^{29}$ M. Iansiti, A. Johnson, W. F. Smith, H. Rogalla, C. J. Lobb, and M. Tinkham, Phys. Rev. Lett. 59, 489 (1987).

${ }^{30}$ R. H. Ono, M. W. Cromar, R. L. Kautz, R. J. Soulen, J. H. Colwell, and W. E. Fogle, IEEE Trans. Magn. MAG-23, 1670 (1987).

${ }^{31}$ R. Frielinghaus, I. E. Batov, M. Weides, H. Kohlstedt, R. Calarco, and Th. Schäpers, Appl. Phys. Lett. 96, 132504 (2016).

${ }^{32}$ J. Xiang, A. Vidan, M. Tinkham, R. M. Westervelt, and C. M. Lieber, Nat. Nanotechnol. 1, 208 (2006).

${ }^{33}$ H. B. Heersche, P. Jarillo-Herrero, J. B. Oostinga, L. M. K. Vandersypen, and A. F. Morpurgo, Nature 446, 56 (2007).

${ }^{34}$ J. P. Cleuziou, W. Wernsdorfer, V. Bouchiat, T. Ondarcuhu, and M. Monthioux, Nat. Nanotechnol. 1, 53 (2006).

${ }^{35}$ M. Veldhorst, M. Snelder, M. Hoek, T. Gang, X. L. Wang, V. K. Guduru, U. Zeitler, W. G. van der Wiel, A. A. Golubov, H. Hilgenkamp, and A. Brinkman, Nat. Mater. 11, 417 (2012).

${ }^{36}$ G. Blatter, M. V. Feigel'man, V. B. Geshkenbein, A. I. Larkin, and V. M. Vinokur, Rev. Mod. Phys. 66, 1125 (1994).

${ }^{37}$ P. Bernstein, J. F. Hamet, M. T. González, and M. Ruibal Acuña, Physica C 455, 1 (2007).

${ }^{38}$ G. Papari, F. Carillo, D. Stornaiuolo, L. Longobardi, F. Beltram, and F. Tafuri, Supercond. Sci. Technol. 25, 035011 (2012).

${ }^{39}$ A. O. Caldeira and A. J. Leggett, Phys. Rev. Lett. 46, 211 (1981).

${ }^{40}$ J. Kurkijarvi, Phys. Rev. B 6, 832 (1972).

${ }^{41}$ T. A. Fulton and L. N. Dunkleberger, Phys. Rev. B 9, 4760 (1974).

${ }^{42}$ L. D. Jackel, W. W. Webb, J. E. Lukens, and S. S. Pei, Phys. Rev. B 9 , 115 (1974).

${ }^{43}$ A. O. Caldeira and A. J. Leggett, Ann. Phys. 149, 374 (1983); A. J. Leggett, J. Phys. Colloq. 39, 1264 (1978)

${ }^{44}$ R. F. Voss and R. A. Webb, Phys. Rev. Lett. 47, 265 (1981); L. D. Jackel, J. P. Gordon, E. L. Hu, R. E. Howard, L. A. Fetter, D. M. Tennant, R. W. Epworth, and J. Kurkijärvi, ibid. 47, 697 (1981); S. WashburnR. A. Webb, R. F. Voss, and S. M. Farris, ibid. 54, 2712 (1985).
${ }^{45}$ M. H. Devoret, J. M. Martinis, and J. Clarke, Phys. Rev. Lett. 55, 1908 (1985); J. M. MartinisM. H. Devoret, and J. Clarke, Phys. Rev. B 35, 4682 (1987).

${ }^{46}$ T. Bauch, F. Lombardi, F. Tafuri, A. Barone, G. Rotoli, P. Delsing, and T. Claeson, Phys. Rev. Lett. 94, 087003 (2005); T. Bauch, T. Lindstrom, F. Tafuri, G. Rotoli, P. Delsing, T. Cleason, and F. Lombardi, Science 311, 57 (2006).

${ }^{47}$ D. Vion, M. Götz, P. Joyez, D. Esteve, and M. H. Devoret, Phys. Rev. Lett. 77, 3435 (1996).

${ }^{48}$ J. M. Kivioja, T. E. Nieminen, J. Claudon, O. Buisson, F. W. J. Hekking, and J. P. Pekola, Phys. Rev. Lett. 94, 247002 (2005).

${ }^{49}$ J. Männik, S. Li, W. Qiu, W. Chen, V. Patel, S. Han, and J. E. Lukens, Phys. Rev. B 71, 220509 (2005).

${ }^{50}$ L. Longobardi, D. Massarotti, G. Rotoli, D. Stornaiuolo, G. Papari, A. Kawakami, G. P. Pepe, A. Barone, and F. Tafuri, Phys. Rev. B 84, 184504 (2011); D. Massarotti, L. Longobardi, L. Galletti, D. Stornaiuolo, D. Montemurro, G. P. Pepe, G. Rotoli, A. Barone, and F. Tafuri, Fiz. Nizk. Temp. 38, 336 (2012) [Low Temp. Phys. 38, 263 (2012)].

${ }^{51}$ L. Longobardi, D. Massarotti, D. Stornaiuolo, L. Galletti, G. Rotoli, F. Lombardi, and F. Tafuri, Phys. Rev. Lett. 109, 050601 (2012).

${ }^{52}$ D. Massarotti, D. Stornaiuolo, P. Lucignano, L. Galletti, D. Born, G. Rotoli, F. Lombardi, L. Longobardi, A. Tagliacozzo, and F. Tafuri, Phys. Rev. B 92, 054501 (2015).

${ }^{53}$ H. Courtois, M. Meschke, J. T. Peltonen, and J. P. Pekola, Phys. Rev. Lett. 101, 067002 (2008).

${ }^{54}$ W. A. Little, Phys. Rev. 156, 396 (1967).

${ }^{55}$ J. S. Langer and V. Ambegaokar, Phys. Rev. 164, 498 (1967); D. E. McCumber and B. I. Halperin, Phys. Rev. B 1, 1054 (1970).

${ }^{56}$ D. S. Golubev and A. D. Zaikin, Phys. Rev. B 78, 144502 (2008).

${ }^{57}$ N. Shah, D. Pekker, and P. M. Goldbart, Phys. Rev. Lett. 101, 207001 (2008).

${ }^{58}$ R. Gross and B. Mayer, Physica C 180, 235 (1991).

${ }^{59}$ E. J. Tarte, G. A. Wagner, R. E. Somekh, F. J. Baudenbacher, P. Berghusi, and J. E. Evetts, IEEE Trans. Appl. Supercond. 7, 3662 (1997); P. F. McBrien, R. H. Hadfield, W. E. Booij, A. Moya, M. G. Blamire, E. J. Tarte, J. Clark, and C. M. Pegrum, IEEE Trans. Appl. Supercond. 9, 3468 (1999).

${ }^{60}$ J. H. T. Ransley, P. F. McBrien, G. Burnell, E. J. Tarte, J. E. Evetts, R. R. Schulz, C. W. Schneider, A. Schmehl, H. Bielenfeldt, H. Hilgenkamp, and J. Mannhart, Phys. Rev. B 70, 104502 (2004).

${ }^{61}$ B. H. Moeckly and R. A. Buhrman, IEEE Trans. Appl. Super-cond. 5, 3414 (1995).

This article was published in English in the original Russian journal. Reproduced here with stylistic changes by AIP Publishing. 